\title{
Reciprocal Data Sharing: Sending Monthly Summary Reports of Syndromic Data to ED
}

\author{
Anthony W. Tam*, Amita Toprani and Robert Mathes \\ New York City Department of Health and Mental Hygiene, Long Island City, NY, USA
}

\section{Objective}

To share monthly summary reports of syndromic data to participating EDs in NYC.

\section{Introduction}

Over several months in 2012, NYC DOHMH syndromic surveillance staff met with directors of all 49 participating EDs in our syndromic system to collect information on their health information systems coding practices [1]. During these interviews, ED directors expressed interest in receiving summary reports of the data they send to the syndromic unit, such as number of ED visits, most common complaints, and temporal and spatial trends. This effort was done to increase communication and cooperation between the syndromic unit and the EDs that provide data to the syndromic system.

\section{Methods}

We contacted a sample of ED directors to inquire about the variable types and visualization formats they would find most helpful in a summary report, as well as the frequency they would like to receive reports. Variables for analysis include date and time of visit, chief complaint, age, sex, residential zip code of the patient, disposition, and mode of arrival. Reports were generated using R and LaTeX.

\section{Results}

The most requested variables included total number of ED visits, top ten chief complaints (stratified by age group, $\leq 17$ years and $\geq 18$ years), average number of visits by day of week and shift, and geographic distribution of visits by zip code. Optimal frequency was determined to be once a month.

We developed a two page report summarizing these major variables, to be created on a monthly basis, compiling and analyzing data from the previous month. Chief complaints were categorized into general categories by a medical epidemiologist, including: injury or accident, cardiac, respiratory, abdominal, complaints involving the head or face, urinary, genital, medication refill or laboratory test, respiratory infections, nonspecific symptoms, and missing or uninformative information. The top ten chief complaints were binned into two tables, one for adults and the other for children. Temporal trends were summarized by average number of visits by 8-hour shift and day of week. A heat map was created to show the geographical distribution of ED patients' residence.

\section{Conclusions}

For health departments that receive syndromic data directly from hospitals, it is critical to establish and keep close relationships with participating EDs. Sharing summary reports with the EDs in our syndromic system is one way to maintain contact with key ED staff, which is important during outbreaks or when we observe changes in data or data quality. Furthermore, regularly sharing data analyses engages EDs with how health departments use their data, making collaborations more likely and improving the success of future reciprocal data sharing.

\section{Keywords}

Syndromic; Surveillance; Emergency; Department; Communication

\section{References}

1. Sell, J. and A. Wong, A Survey of Data Recording Procedures at New York City Emergency Departments. Online J Public Health Inform, 2013. 5(1): p. e114.

\section{*Anthony W. Tam}

E-mail: atam@health.nyc.gov 